ARTIGO

\title{
Aprendizagem autorregulada do desenho artístico em estudantes universitários*
}

\author{
Luiz Gustavo Lima Freire' (D) \\ António Manuel Duarte' (D
}

RESUMO

Este estudo teve como objetivo verificar a possível existência de duas formas de aprendizagem do desenho artístico: hetero e autorregulada. Pretendeu-se ainda explorar as estratégias de autorregulação possivelmente utilizadas para esse fim. Foram assim entrevistados dezesseis estudantes universitários de uma disciplina de desenho, com base em um roteiro. As respostas, submetidas a uma análise de conteúdo temática, evidenciaram, para além de uma representação indefinida sobre as estratégias que se utilizam, a existência de uma aprendizagem do desenho tanto heterorregulada como autorregulada, que por sua vez envolve uma diversidade de estratégias (Autoavaliação; Organização e transformação; Planificação e formulação de metas; Busca de informação; Supervisão e tomada de registros; Estruturação do ambiente; Revisão e memorização; Procura de assistência; Autocontrole).

\section{PALAVRAS-CHAVE}

aprendizagem; arte; autorregulação; desenho; estratégias de aprendizagem.

*Investigação desenvolvida no âmbito de um doutoramento financiado pela Fundação para a Ciência e a Tecnologia (FCT) por meio da Bolsa SFRH/BD/62430/2009.

'Universidade de Lisboa, Lisboa, Portugal. 


\section{SELF-REGULATED LEARNING OF ARTISTIC DRAWING IN UNIVERSITY STUDENTS}

\section{ABSTRACT}

This study aimed to verify the possible existence of two forms of learning artistic drawing: hetero and self-regulated. It also sought to explore self-regulation strategies possibly used for that goal. Based on a script, sixteen university students from a drawing course were therefore interviewed. The answers, submitted to a thematic analysis, showed, in addition to an indefinite representation on the used strategies, the existence of either a hetero or a self-regulated learning that, in turn, involves a diversity of strategies (i.e., Self-evaluation, Organization and transformation; Planning and formulation of goals; Information search; Supervision and recordkeeping; Environmental structuring; Reviewing and memorization; Seeking assistance; Self-control).

\section{KEYWORDS}

art; drawing; learning; learning strategies; self-regulation.

\section{APRENDIZAJE AUTORREGULADA DEL DIBUJO ARTÍSTICO EN ESTUDIANTES UNIVERSITARIOS}

\section{RESUMEN}

Este estudio tuvo como objetivo verificar la posible existencia de dos formas de aprendizaje del dibujo artístico: hetero y autorregulado. También se pretendió explorar las estrategias autorregulatorias posiblemente utilizadas para ello. Por lo tanto, fueron entrevistados dieciséis estudiantes universitarios de una disciplina de diseño, con base en un guión. Las respuestas, sometidas a un análisis de contenido temático, evidenciaron, además de una representación indefinida sobre las estrategias que se utilizan, la existencia de un aprendizaje del diseño tanto heteroregulado como autorregulado, que a su vez implica una diversidad de estrategias (Autoevaluación; Organización y transformación; Planificación y formulación de metas; Búsqueda de información; Supervisión y toma de registros; Estructuración del ambiente; Revisión y memorización; Búsqueda de asistencia; Autocontrol).

PALABRAS CLAVE

aprendizaje; arte; autorregulación; diseño; estrategias de aprendizaje. 


\section{INTRODUÇÃO}

A investigação em psicologia educacional tem oferecido evidências de que, paralelamente a outras variáveis, a competência dos estudantes em autorregularem a sua aprendizagem se associa significativamente com o seu sucesso acadêmico (Silva e Sá 1997; Silva et al., 2004). No mesmo sentido, tem sido constatado que a promoção daquela competência pode contribuir para reduzir significativamente o fracasso traduzido na repetência e evasão escolares (Ribeiro, 2013).

Quando aplicada à aprendizagem em contexto educacional, a autorregulação implica um processo autodirecionado, por meio do qual os estudantes transformam suas habilidades mentais em capacidades acadêmicas (Zimmerman, 1998; Zimmerman e Labuhn, 2012) utilizando estratégias instrumentais dirigidas à aquisição de informação ou competência (Zimmerman, 1989). Nesse contexto, a autorregulação implica igualmente um feedback auto-orientado durante a aprendizagem, que envolve conhecimentos sobre como e por que escolher uma estratégia específica, avaliações sobre as direções que são tomadas para realizar os objetivos e eventuais mudanças da estratégia selecionada (Bandura, 2008). Ao exercer esse controle, o estudante adota padrões internos, monitora seus comportamentos e utiliza incentivos motivacionais para mobilizar e sustentar seus esforços e para cumprir o que se havia proposto (Bandura, 2008; Zimmerman e Martinez-Pons, 1986). A autorregulação da aprendizagem envolve, assim, uma grande diversidade de processos: crenças autorreferenciadas ou relacionadas ao meio (Hla e Phyu, 2011); atribuição de valor às atividades de aprendizagem (Bandura, 2008; Deci e Ryan, 2012; González, 2015; Pintrich, 2000); elementos motivacionais compreendidos na inicialização das tarefas, assim como decisões volitivas, de escolha de tarefas, de determinação do nível de empenho e de manutenção e persistência (Silva e Sá 1997; Silva et al., 2004); estratégias cognitivas de processamento (e.g., aquisição, organização e retenção) da informação (Daura, 2015; Hinojosa e Sanmartí, 2015; Monereo et al., 1995; Roux e González, 2015; Sepulveda-Vildosola, Carada-Legaria e Reyes-Lagunes, 2015); comportamentos de execução de procedimentos ou ações orientados para os objetivos (Silva e Sá 1997; Silva et al., 2004); e estratégias metacognitivas, de planejamento, monitorização e autoavaliação dos processos de aprendizagem (Paris e Winograd, 1990; Ribeiro, 2013; Simão, 2004; Wagener, 2015).

Mais especificamente, a autorregulação da aprendizagem constitui um processo cíclico, que se desenvolve em três fases interdependentes e potencialmente concomitantes (Zimmerman, 2000):

1. antecipação: determinação de objetivos e seleção de estratégias para alcançá-los;

2. desempenho: execução, automonitorização e autocontrole cognitivo e motivacional;

3. autorreflexão: avaliação da produção acadêmica por meio da sua comparação com os objetivos.

Para avaliar a autorregulação da aprendizagem em contextos naturalísticos, Zimmerman e Martinez-Pons (1986) desenvolveram um instrumento, o 
Self-Regulated Learning Interview Schedule (SRLIS), que a considera um processo motivacional, cognitivo, comportamental e metacognitivo, acessível no relato direto de estudantes. Esse instrumento permite a realização de uma entrevista estruturada, na qual se questiona os estudantes sobre as estratégias que utilizam em diferentes situações de aprendizagem, no contexto interno e externo da sala de aula. Aplicando o SRLIS a estudantes do ensino secundário, Zimmerman e Martinez-Pons (1986) detectaram, por meio de uma análise dedutiva, a existência de catorze tipos de estratégias de aprendizagem autorregulada, derivadas da teoria:

1. autoavaliação;

2. organização e transformação (de materiais instrucionais);

3. planificação e formulação de metas;

4. busca de informação;

5. supervisão e tomada de registros;

6. estruturação do ambiente;

7. autoconsequências;

8. revisão e memorização;

9 a 11. procura de assistência social:

9. dos pares; 10. dos professores; 11. dos adultos;

12 a 14. revisão de registros:

12. de testes; 13. de apontamentos; 14 . de livros.

Para além dessas estratégias de aprendizagem autorregulada, os autores observaram, nesse mesmo estudo, um décimo quinto padrão de aprendizagem, então designado de Outro, que se refere a uma aprendizagem heterorregulada por outras pessoas, como os professores e os pais. Com base no SRLIS, corroborando os seus resultados originais, diversos estudos posteriores, alguns deles no contexto dos países de língua portuguesa, têm desenvolvido e utilizado instrumentos de avaliação sensíveis à aprendizagem autorregulada (Boruchovitch, 1995; Costa e Boruchovitch, 2004,2010a, 2010b; Encarnacao e Vázquez-Bernal, 2013; Lee e Jiar, 2014; Rosário et al., 2005; Teixeira, 2004; Teixeira e Alliprandini, 2013).

No entanto, a aprendizagem autorregulada tem vindo a ser, sobretudo, estudada no contexto das áreas de domínio (conceitual) da linguagem (Sousa, 2015), da matemática (Becker, 2016) e das ciências (Corrêa e Correia, 2017), sendo igualmente necessária a sua conceitualização e investigação na área da aprendizagem da arte.

\section{APRENDIZAGEM AUTORREGULA DA ARTE}

A aplicação da perspectiva da autorregulação à aprendizagem da arte sugere que o estudante que experiencia uma aprendizagem artística integradora se interessa e persiste nas tarefas que enfrenta, prova as suas capacidades, questiona, avalia e modifica a sua forma de enfrentar as tarefas, toma consciência de suas debilidades, recorre a novos (mais) esforços em momentos críticos da sua aprendizagem e atribui o êxito ao desempenho eficaz (Catterall, 2005; Kaplan, 2009; Soto e Macías, 2011). Paralelamente, tal como apontado por Eisner (2002), a expressão artística 
pressupõe o estabelecimento de um objetivo anterior à ação sobre um material, que assim envolverá, na aprendizagem das artes plásticas, o desenvolvimento da planificação (e.g., seleção de cores, texturas e materiais), da execução (e.g., aplicação imaginativa de técnicas) e da avaliação (e.g, comparação do resultado com o objetivo ou o modelo). Apesar de escassa, tem sido realizada alguma investigação sobre a autorregulação no domínio específico da aprendizagem da arte.

Com enfoque na aprendizagem da música, Araújo, Cavalcanti e Figueiredo (2010) detectaram a maior eficiência, em constraste ao desempenho de atividades repetitivas, de uma prática musical que envolve planejamento, estabelecimento de metas e determinação de estratégias adequadas, que possibilitem ao instrumentista a oportunidade de alcançar seus objetivos. Para além disso, constataram importantes efeitos nos resultados artísticos da confiança que o estudante tem em sua capacidade de desempenho, da concentração e persistência na prática musical e da satisfação com a tarefa.

Igualmente na área da música, Gusmão (2011) identificou uma relação entre o desempenho em disciplinas de teoria e percepção musical e a utilização de processos autorregulatórios, tais como o estabelecimento de prioridades, a gestão do tempo, a utilização de padrões internos de avaliação, a elevada autoeficácia, a atribuição de valor às tarefas, o esforço e a persistência. Em contraposição, constatou uma associação entre problemas no desempenho e diversas falhas nos processos autorregulatórios, tais como a reduzida autoeficácia, a ausência de estabelecimento de objetivos, que permitiriam um automonitoramento e autoavaliação mais eficientes, e a existência de uma autoavaliação do desempenho e do progresso exclusivamente baseada em padrões externos predefinidos, tais como as classificações necessárias à aprovação.

Por seu lado, Winner e Hetland (2001), em estudo sobre a aprendizagem das artes visuais em estudantes universitários, apuraram a possibilidade de esta se realizar nos termos da autorregulação (Zimmerman, 1989, 2000), na medida em que se observou que aqueles estudantes podiam aprender: a desenvolver uma técnica (como e quando utilizar instrumentos e materiais); a persistir nas tarefas (atender a projetos durante um longo período); a imaginar (gerar imagens mentais que ajudem a antecipar e orientar as obras); a expressar-se (desenvolver uma visão e um significado pessoal); a observar (notar as qualidades dos objetos, tais como detalhes, brilhos, texturas, cores, estilos etc.); a refletir (sobre suas obras e as dos demais, questionando e explicando suas intenções e decisões durante o processo de elaboração); a experimentar (ir além do que haviam feito, correndo riscos); e a compreender conteúdo (sobre a história da arte e como ela se relaciona com o mundo).

Em termos mais gerais, Soto e Macías (2011) revelaram que uma educação artística integrada tem o potencial de desenvolver a autonomia e a reflexão dos estudantes, particularmente promovendo a expressão de suas sensações, pensamentos, criatividade e emoções, quando enriquecida com os fundamentos da aprendizagem autorregulada.

Finalmente, na área particular da aprendizagem do desenho, a investigação sobre a autorregulação parece praticamente inexistente. Uma exceção é Padilla (2007), que, salientando o processo perceptivo-motor implicado na aprendizagem 
do desenho, refere que o estudante avança, aqui, fragmentariamente, mediante tentativas, erros e ajustes subordinados à execução de procedimentos para alcançar os objetivos, à comprovação de sua prossecução e a eventuais reordenamentos, caso aqueles não tenham sido atendidos.

Considerando a escassez de investigação sobre o tema, o objetivo deste estudo foi o de averiguar a possível replicação na aprendizagem do desenho artístico de observação da dicotomia observada por Zimmerman e Martinez-Pons (1986) entre a aprendizagem heterorregulada (i.e., controlada por outras pessoas, que não o estudante que aprende) e a aprendizagem autorregulada (i.e., controlada pelo estudante que aprende), no nível da aprendizagem escolar indiferenciada. Pretendeu-se ainda verificar a possível replicação e a especificidade das estratégias de aprendizagem autorregulada identificadas por aqueles autores, mediante o SRLIS, no âmbito da aprendizagem do desenho. Esse objetivo foi paralelo ao objetivo de explorar ainda outras estratégias de autorregulação, eventualmente envolvidas na aprendizagem particular do desenho, assim como de explorar a representatividade das estratégias encontradas.

\section{MÉTODO}

\section{PARTICIPANTES}

Participaram no estudo 16 estudantes universitários, 9 (56,25\%) do sexo masculino e $7(43,75 \%)$ do sexo feminino, com idade que variava entre 18 e 28 $(\mathrm{M}=22)$ de vários cursos: matemática $(18,75 \%)$; ciências biológicas $(68,75 \%)$; e letras $(12,50 \%)$.

A dimensão da amostra seguiu um critério de saturação das categorias detectadas, isto é, cessou-se de entrevistar mais estudantes quando da análise do discurso do último entrevistado não resultaram novas categorias.

A amostragem foi por conveniência, de acordo com a disponibilidade dos estudantes que estavam cursando uma disciplina de desenho artístico, selecionados com base no pressuposto de que a sua experiência lhes permitia uma articulação das estratégias envolvidas na aprendizagem do desenho. Esses estudantes cursavam uma disciplina optativa livre de desenho artístico de observação de uma universidade pública brasileira. De acordo com o currículo da disciplina, a sua finalidade é desenvolver a aprendizagem dos elementos básicos necessários à promoção do desenho de criação, estimulando a aplicação correta e sintética da forma, do volume, da proporção, da cor e do equilíbrio, em uma composição e habilitando o aluno a captar mentalmente uma imagem e a transpor para o material. Para tanto, o docente fornece exercícios de transposição da imagem para o papel e técnicas de desenho. Entre os conteúdos, destacam-se o corpo do desenhista (a função da respiração e do olho, a memória corporal, equilíbrio corporal/gráfico e membros), o desenho como manifestação criativa (geometrização, sombra, perspectiva, sentimento, deformações dos objetos no desenho e criatividade) e técnicas de desenho. 


\section{PROCEDIMENTO DE RECOLHA DE DADOS}

$\mathrm{O}$ acesso aos dados relativos à utilização das estratégias pessoais implicadas na aprendizagem do desenho se deu no marco de uma entrevista semiestruturada. A entrevista teve como apoio um roteiro composto de catorze questões (Anexo) baseadas no SRLIS de Zimmerman e Martinez-Pons (1986), instrumento traduzido e adaptado para o português por Boruchovitch (1995), apresentado na Introdução.

Com a finalidade de testar o grau de clareza e de entendimento dos objetivos das questões, procedeu-se a uma aplicação piloto a um estudante e à análise do docente da disciplina de desenho artístico em questão.

$O$ roteiro de entrevista contempla, assim, catorze questões orientadas para os seguintes cenários/temas (dimensões) das estratégias envolvidas na aprendizagem do desenho:

1. sala de aula;

2. compreensão;

3. planejamento;

4. casa;

5. preparação para a avaliação;

6. motivação para fazer o dever de casa;

7. memorização;

8. administração do tempo;

9. realização de provas;

10. correção de desempenho;

11. motivação para a aprendizagem;

12. organização do ambiente de aprendizagem;

13. autoavaliação do resultado;

14. autoavaliação da atividade.

A recolha de dados foi realizada sob consentimento informado da coordenação do departamento responsável pela disciplina em questão, que se responsabilizou pela concordância do estudo ao código de ética da universidade, pelo docente da disciplina e pelos participantes, a quem foi assegurada a confidencialidade dos dados.

As entrevistas foram realizadas em um local que garantia privacidade, no recinto da universidade, tendo sido gravadas em áudio e posteriormente transcritas.

\section{PROCEDIMENTO DE ANÁLISE DE DADOS}

Os dados recolhidos foram inicialmente sujeitos a uma análise de conteúdo temática que envolveu três fases:

1. segmentação das respostas em unidades a categorizar;

2. categorização das unidades segmentadas;

3. exploração das categorias encontradas.

A segmentação das respostas foi dedutiva, considerando o seu contexto global e utilizando um critério de segmentação por unidades temáticas (Flores, 1994): 
atendendo-se às dimensões patentes no roteiro de entrevista, ou seja, sempre que uma parte do discurso aludia a uma das dimensões do roteiro (cenários/temas das estratégias implicadas na aprendizagem do desenho) era considerado (segmentado) como uma unidade de análise a ser categorizada posteriormente. A segmentação das respostas foi realizada independentemente por dois analistas, com um grau de acordo de $96,5 \% .{ }^{1}$ Posteriormente, os casos de discordância foram resolvidos por discussão entre eles.

A categorização das unidades segmentadas foi do tipo intermédio, ou seja, as unidades foram inicialmente categorizadas de acordo com o sistema de quinze estratégias identificadas por Zimmermam e Martinez-Pons (1986) referido na Introdução, mas o sistema foi sendo enriquecido com categorias emergentes, tal como sugerido por Miles e Huberman (1994). O sistema resultante, que coincide com o sistema de categorias apresentado na seção Resultados, foi depois aplicado independentemente pelos dois analistas, para categorização das unidades segmentadas. O grau de acordo entre os analistas variou entre $85,7 \%$ e $100 \%$ para as diferentes dimensões, com uma média de $95.7 \%$. Posteriormente, foi também calculado o acordo intra-analista $(85,7 \%)$, com base na comparação entre a primeira e uma segunda categorização, por um dos analistas, de 14,7\% dos segmentos de resposta.

Finalmente, procedeu-se à exploração das categorias identificadas, analisando a sua representatividade (frequência relativa) na amostra de participantes inquirida. Para tal, a presença de cada categoria no discurso de um participante foi contada como uma incidência da respectiva categoria na amostra, independentemente do número de vezes em que está presente naquele mesmo discurso.

\section{RESULTADOS}

Apresenta-se, em seguida, cada uma das categorias resultante da análise às entrevistas realizadas, indicando a sua frequência relativa na amostra de participantes (\%), o seu significado e dois excertos de respostas que a ilustra.

VAGA

A categoria Vaga (apresentada por $56,5 \%$ dos participantes) reflete uma percepção indefinida ou pouco elaborada das estratégias pessoais implicadas na aprendizagem do desenho.

[...] sempre gostei do setor de arte em geral [...] é uma coisa que me interessa $[\ldots]$.

$[\ldots]$ botando a mão na massa, é o que mais ajuda a desenhar [...].

1 Para todos os cálculos de coeficiente de acordo entre os analistas, foi utilizada a fórmula sugerida por Bakeman e Gottman (1986): $\mathrm{PA}=(\mathrm{Na} /(\mathrm{Na}+\mathrm{Nd})) 100$, em que "PA" é a percentagem de acordo; "Na" é a frequência de acordo e "Nd" é a frequência dos desacordos. 


\section{OUTRO}

A categoria Outro (apresentada por 37,5\% dos participantes) equivale a uma aprendizagem heterorregulada do desenho, ou seja, nesse caso a aprendizagem do desenho parece ser determinada por outras pessoas (e.g., professores, pais) ou pelo contexto no qual o estudante está inserido.

$[\ldots]$ faço $[\ldots]$ o que me foi mandado fazer $[\ldots]$.

[...] trago na outra semana para ele [o professor] [...] me dizer o que é que eu tenho que melhorar $[\ldots]$.

\section{ESTRUTURAÇÃO DO AMBIENTE}

A categoria Estruturação do ambiente (apresentada por 100\% dos participantes) refere-se a uma estratégia de selecão ou organização do ambiente físico da atividade do desenho.

[...] procuro um local [...] apropriado [...] confortável, iluminado, arejado [...] é mais propício [...] do que um local que você vai estar $[. .$.$] incomodado [...].$

$[\ldots]$ o ambiente tem que ser $[\ldots]$ favorável $[. .$.$] que não tenha muita gente [. .$. na minha casa $[\ldots]$ desenho no meu quarto [...] num lugar mais tranquilo [...] não gosto de ir para um ambiente [...] carregado, com poluição visual [...] barulho, porque eu não me concentro ou com [...] pessoas falando [...].

\section{AUTOAVALIAÇÃO}

A categoria Autoavaliação (apresentada por 100\% dos participantes) equivale a uma estratégia de autoavaliação da qualidade ou do progresso da atividade do desenho.

[...] é uma harmonia dos sentidos [...] saber ver e sentir aquele desenho, se está saindo bem $[\ldots][\mathrm{ou}]$ mal [...] ter um olhar crítico [...] ser um autocorretor $[\ldots]$ você mesmo se corrigir [...] se avaliar no desenho [...] em que ponto está bom $[\ldots][\mathrm{ou}]$ ruim $[\ldots]$.

[...] boto em comparação com outros trabalhos que eu já tenha feito [...] uma técnica tipo degradê $[\ldots]$ observo como eu comecei fazendo [...] e [...] onde $[\ldots]$ estou $[\ldots]$.

\section{PROCURA DE ASSISTÊNCIA}

A categoria Procura de assistência (apresentada por 93,7\% dos participantes) equivale à estratégia de solicitação de ajuda a outras pessoas na e para a atividade 
de desenho, compreendendo três variantes. A primeira variante, designada de professor (em 93,7\% dos participantes), refere-se à estratégia de soliticação de ajuda ao professor.

$[\ldots]$ peço ajuda ao professor $[\ldots]$ que me oriente $[\ldots]$.

[...] peço ajuda para ele [o professor] de que forma eu posso fazer para que o desenho saia melhor? [...].

A segunda variante, denominada de colegas (em 43,7\% dos participantes), reconhece a existência de uma estratégia de solicitação de ajuda aos colegas.

$[\ldots]$ recorro à ajuda $[\ldots]$ do colega $[\ldots]$.

[...] peço ajuda aos meus amigos $[\ldots]$.

A terceira variante, designada de pessoas indiferenciadas (em 50\% dos participantes), refere-se a uma estratégia de solicitação de ajuda a indivíduos não especificados.

$[\ldots]$ peço ajuda $[\ldots]$ a quem for $[\ldots]$.

[...] eu sempre mostro para as pessoas próximas [...].

\section{METAS E PLANIFICAÇÃO}

A categoria Metas e planificação (apresentada por 93,7\% dos participantes) refere-se a uma estratégia de estabelecimento de metas e planificação da atividade do desenho.

[...] tem que ser planejado, os horários [...] administrar o tempo [...] há um momento no meu dia [...] meus horários, que é voltado para isso, não só a feitura do desenho $[\ldots]$ mas a leitura de textos relacionados $[\ldots]$ ao desenho $[\ldots]$.

[...] tem que ter o planejamento do seu horário [...] dedicar um tempo [...] proporcional $[. .$.$] à demanda [. .$.$] eu preciso de quatro horas por semana em$ casa para desenhar $[\ldots]$.

\section{AUTOCONTROLE}

A categoria Autocontrole (apresentada por 93,7\% dos participantes) equivale a estratégias de autocontrole da atividade de desenhar, paralelas ao seu desempenho.

$[\ldots]$ quando estou fazendo um desenho $[\ldots]$ não me preocupo $[\ldots]$ com o que eu vou fazer daqui a pouco $[\ldots]$ as provas $[\ldots]$ as tarefas $[\ldots]$ eu me desligo de 
tudo $[. .$.$] para me concentrar só naquela atividade, aí eu [. .$.$] consigo um de-$ sempenho maior $[\ldots]$.

[...] concentração [...] se entregar mais àquilo e prestar mais atenção no que você está fazendo [...].

\section{REVISÃO E MEMORIZAÇÃO}

A categoria Revisão e memorização por repetição (apresentada por 100\% dos participantes) corresponde a uma estratégia de memorização de procedimentos de desenho por meio da prática.

$[\ldots]$ vendo $[\ldots]$ fazendo $[. .$.$] porque minha memoria é péssima, eu tenho que$ fazer, ficar treinando para [...] ir memorizando [...].

[...] a partir da repetição, você desenha numa aula [...] na outra [...].

\section{ORGANIZAÇÃO E TRANSFORMAÇÃO}

A categoria Organização e transformação (apresentada por 75\% dos participantes) diz respeito a uma estratégia de organização da atividade do desenho.

[...] tem que traçar um plano, um projeto, você não vai começar a desenhar aleatoriamente $[\ldots]$.

[...] prefiro preparar tudo e depois [...] começar a andar com [...] facilidade, do que ir andando sem $[. .$.$] certeza de como [\ldots]$ vai ficar $[\ldots]$.

\section{BUSCA DE INFORMAÇÃO}

A categoria Busca de informaşão (apresentada por $43,7 \%$ dos participantes) equivale a uma estratégia de busca de informação adicional sobre a atividade do desenho, tal como informação sobre temas, técnicas e estilos de desenho.

$[\ldots]$ recorro à internet, porque $[\ldots]$ pode-se achar técnicas $[\ldots]$.

$[\ldots]$ eu mesmo fui procurar $[\ldots]$ pegar fotos fáceis de desenhar $[\ldots]$ de revista $[\ldots]$ que tenham os traços bem marcantes $[\ldots]$.

\section{SUPERVISÃO E TOMADA DE REGISTROS}

A categoria Supervisão e tomada de registros (apresentada por 18,7\% dos participantes) equivale a uma estratégia de registro de informação sobre a atividade de desenho, tal como o registro de técnicas de desenho. 
$[\ldots]$ eu anoto $[\ldots]$ métodos que o professor fala na sala para não esquecer $[\ldots]$ coisas de medida [...].

[...] eu tento ler os conceitos que [...] anoto, faço anotações sobre o que o professor fala $[\ldots]$.

\section{DISCUSSÃO}

Os resultados da análise efetuada sobre o processo de aprendizagem de desenho dos participantes inquiridos replicam, em sua generalidade, a realidade observada por Zimmerman e Martinez-Pons (1986) (o referencial de avaliação mencionado na Introdução), no que diz respeito à aprendizagem escolar indiferenciada. Os resultados sugerem que, tal como para essa aprendizagem, sobretudo de índole conceitual, a aprendizagem do desenho, especialmente técnica e expressiva, pode ocorrer igualmente de forma hetero ou autorregulada, embora neste último caso com especificidades próprias. Para além de uma possível razão relacionada com diferenças individuais, essa dicotomia reflete, talvez, uma natural tensão entre a necessária dependência de aprendentes iniciados em relação ao contexto social (i.e., professor, pais), em que uma aprendizagem técnica se verifica, e a igualmente necessária independência que a mesma aprendizagem requer e o nível de desenvolvimento que possibilita.

A categoria Vaga (apresentada por 56,5\% dos participantes), que reflete uma percepção indefinida ou pouco elaborada das estratégias de autorregulação que se utiliza para a aprendizagem do desenho artístico de observação, poderá ser explicada, por um lado, pela possível ausência de uma reflexão anterior, por parte dos participantes, acerca da utilização dessas mesmas estratégias, por outro, pela inexistência (ou carência) desse tipo de estratégias no repertório por eles utilizado.

A categoria Outro exprime uma aprendizagem heterorregulada do desenho, que replica outra, com o mesmo nome, cuja presença Zimmerman e Martinez-Pons (1986) observaram para a aprendizagem em geral. A sua presença, minoritária, mas não negligenciável (37,5\%), pode ser explicada, quer por eventuais características pessoais (e.g,. personalidade mais dependente), quer pelo fato de a aprendizagem do desenho parecer contar, especialmente na fase de iniciação, com a introdução de competências técnicas em um contexto social mais fechado (e.g., exercícios de desenho fornecidos e monitorizados pelo professor).

As restantes categorias detectadas replicam todas as outras estratégias de aprendizagem autorregulada observadas (e designadas com o mesmo nome) por Zimmerman e Martinez-Pons (1986), com exceção das estratégias de Autoconsequências e de Revisão de registros, aqui não encontradas, e da categoria aqui detectada de Autocontrole, não observada por aqueles autores.

A categoria Estruturação do ambiente (i.e., seleção ou organização do ambiente físico da atividade do desenho), muito expressiva na amostra (100\%), confirma a noção de Zimmerman (1989) de que a aprendizagem autorregulada envolve não 
só o autocontrole dos comportamentos, mas também o controle do ambiente de aprendizagem. Esse dado pode estar também relacionado ao próprio aspecto instrumental da aprendizagem do desenho, que implica o manejo de materiais ou ferramentas concretas. Essa estratégia é também consonante com a importância que a estruturação do ambiente físico de trabalho parece ter na atividade criativa de muitos artistas (Currey, 2013).

A categoria Autoavaliação (da qualidade ou progresso da atividade do desenho), com presença muito significativa (100\%), testemunha a existência da fase autorregulatória da autorreflexão (Bandura, 1986; Horta, 2010; Rosário et al., 2010; Zimmerman, 1998; Zimmerman e Martinez-Pons, 1986), também no caso da aprendizagem do desenho. Efetivamente, considerando que essa aprendizagem parece decorrer da contínua atividade de desenhar, ela possivelmente envolverá um constante confronto autocrítico e autocorretor do estudante com os seus desenhos. Em contrapartida, essa estratégia também não será alheia ao fato de o processo criativo parecer consistir em uma dinâmica que envolve a relação entre processos generativos e processos exploratórios, na qual está inserida a avaliação do que se produz (Finke, Ward e Smith, 1992).

A categoria Procura de assistência social (solicitação de ajuda a colegas, professores e outras pessoas), muito expressiva na amostra (93,7\%), reflete talvez a necessidade de se gerir o necessário apoio social requerido por uma aprendizagem técnica, como será a do desenho, especialmente na fase de iniciação. $\mathrm{O}$ recurso intencional a outras pessoas poderá ser igualmente reflexo do ambiente típico da aprendizagem do desenho (e.g., um ateliê), onde se pode circular e interagir mais facilmente com colegas e professores. Para além disso, sabemos que a criação artística (e.g., em design e arquitetura) pode ocorrer não só individualmente, mas no contexto da colaboração entre artistas (Lawson, 2003). Essa categoria reforça, assim, a noção sociocognitiva de que a autorregulação da aprendizagem não é um processo necessariamente associal, podendo envolver a interação ativa com os outros (Soto e Macías, 2011; Zimmerman e Martinez-Pons, 1986). O professor é referido como a maior fonte de assistência social procurada $(93,7 \%)$, possivelmente pela competência técnica reconhecida. Paralelamente, a referência à utilização dos colegas como fonte de assistência $(43,7 \%)$ tem provavelmente como razão, quer a proximidade física e afetiva mantida com eles, quer a interação cooperativa que com eles se estabelecerá, em virtude da aprendizagem do desenho ser percebida como um objetivo comum. Para além disso, também aqui, como sugerido por Zimmerman (2000), o feedback social parece estar sendo igualmente utilizado como critério de autoavaliação e de correção de desempenho, considerando que a categoria Procura de assistência social, por vezes, emerge de questões orientadas à autoavaliação do processo e dos resultados da aprendizagem do desenho.

A categoria Metas e planificação, que manifesta a preocupação em gerir a aprendizagem do desenho, muito significativa na amostra (93,7\%), poderá traduzir a necessidade dos estudantes em organizarem essa aprendizagem em função de recursos compartidos com outras atividades (e.g., um tempo que tem de ser repartido por uma diversidade de disciplinas e de tarefas típicas da sua fase de vida). A importância da gestão do tempo de aprendizagem do desenho é, ainda, consistente com 
a relevância que o controle do tempo pessoal aparenta ter no trabalho de muitos artistas (Currey, 2013).

A categoria Autocontrole (93,7\%) não coincide com nenhuma das estratégias de aprendizagem autorregulada encontradas por Zimmerman e Martinez-Pons (1986). No entanto, essa categoria parece refletir estratégias de autocontrole do desempenho tipicamente existentes na fase autorregulatória de Desempenho (execução, automonitorização e autocontrole cognitivo e motivacional por mobilização de forças pessoais ou recursos do meio para atingir os objetivos pretendidos). O autocontrole da atividade de desenho pode hipoteticamente ser explicado pelo fato de esta (com a apreensão e a representação de uma forma em um todo que se consitui a partir de diferentes partes) possivelmente envolver a necessidade de um comprometimento intensivo e exclusivo. Esse autocontrole poderá estar igualmente associado à necessidade sentida por muitos artistas em controlarem (e.g., por meio de rotina de trabalho) potenciais distrações ao seu desempenho (Currey, 2013).

A categoria Revisão e memorização, muito expressiva na amostra (100\%), sugere a utilização de uma estratégia de memorização dos procedimentos de desenho por meio da prática. Essa estratégia pode ser explicada pelo fato de a atividade de desenho exigir a coordenação de movimentos muito precisos, cujo desenvolvimento se baseia na criação de hábitos que constituem, segundo Kaplan (2009), reações automáticas e estereotipadas, adquiridas pela repetição perante situações idênticas. São esses mesmos hábitos que, reorganizados, estão na base da utilização de técnicas para cuja aprendizagem o exercício continuado é muito importante. A repetição adquire, aqui, enorme relevância no processo de memorização, porque, além de favorecer a retenção, como acontece na aprendizagem do tipo conceitual, proporciona também a criação de hábitos e de habilidades motoras necessários para desenhar.

A categoria Organização e transformação, com uma representatividade considerável (75\%), poderá refletir a necessidade frequentemente encontrada no desenho, e, aliás, na criação de muitas obras de arte, de realizar esboços. Esses esboços constituem traços preparatórios (ou de estudo) frequentemente necessários à elevação dos planos e à projeção dos corpos, muitas vezes realizados pela associação dos modelos a figuras geométricas. Os esboços facilitam a elaboração de ideias, assim como o manuseio, a modificabilidade e a adaptação do desenho em virtude do desprendimento e da flexibilidade que envolvem.

A categoria Busca de informação (sobre a atividade de desenho), aqui relativamente menos representada (43,7\%), constitui indício da replicação, nesse contexto, de uma estratégia observada não só no estudo de Zimmerman e Martinez-Pons (1986), mas também por outros estudos posteriores (Costa e Boruchovitch, 2010b; Encarnacao e Vázquez-Bernal, 2013). Nesse caso, a procura de informação adicional sobre o conteúdo em aprendizagem (e.g., temas, técnicas ou estilos de desenho) compreende-se, quer em virtude da diversidade de informação existente sobre uma atividade sofisticada como a do desenho, quer da necessidade de aprofundamento da aprendizagem, que se começa a exprimir de forma mais significativa no nível do ensino superior (Biggs, 1987).

A categoria Supervisão e tomada de registros, de presença minoritária (18,7\%), que manifesta uma estratégia de registro de informação na atividade de desenho, 
atesta alguma relevância do registro (e.g., de técnicas) na aprendizagem do desenho. No entanto, a reduzida incidência dessa estratégia pode ser lida à luz da natureza $\mathrm{da}$ aprendizagem do desenho, preferentemente de procedimentos (e.g., destrezas psicomotoras) que de conteúdos declarativos, mais passíveis de registro. Essa reduzida incidência é ainda coerente com a alta incidência da estratégia Procura de assistência social. Com efeito, na fase inicial da aprendizagem do desenho, a orientação do professor será mais essencial, corrigindo os erros em tempo real (ao vivo) e estimulando o aluno, até que o movimento de suas mãos se torne calibrado e consiga reproduzir o que foi percebido, de forma relativamente automática. Como refere Kaplan (2009), é particularmente difícil aprender habilidades motoras conceitualmente.

Em suma, no caso da aprendizagem do desenho aqui investigado, para além do equivalente a uma aprendizagem heterorregulada, replicaram-se os equivalentes a todas as estratégias de aprendizagem autorregulada identificadas por Zimmerman e Martinez-Pons (1986), com exeção dos equivalentes às estratégias Autoconsequências (i.e., autoadministração de recompensas ou punições para os sucessos ou fracassos escolares) e Revisão de registros (i.e., revisão de testes, apontamentos ou livros). A ausência de um equivalente à estratégia de Autoconsequências pode, talvez, ser explicada em virtude da observação anedótica de que os estudantes inquiridos apresentavam uma elevada motivação intrínseca para a aprendizagem do desenho, com base no prazer que dela retiravam. Lembremos que essa aprendizagem se dava no contexto de uma disciplina optativa ou de escolha livre e que os estudantes eram oriundos de áreas mais "duras" (i.e., Matemática, Ciências Biológicas e Letras). Paralelamente, a ausência de um equivalente à estratégia de Revisão de registros pode, talvez, ser explicada igualmente pela natureza da aprendizagem do desenho, mais de procedimentos que de conteúdo declarativo, que é mais passível de registro.

Finalmente, algumas das estratégias de autorregulação apresentadas pelos entrevistados sugerem que o caráter cíclico da autorregulação da aprendizagem, em três fases interdependentes, tal como proposto por Zimmerman $(1989,2000)$, igualmente se reflete no caso particular da aprendizagem do desenho. Com efeito, para além da estratégia de Autoavaliação, representativa da fase de Autorreflexão, os estudantes entrevistados também apresentaram a estratégia de Planificação e formulação de metas, representativa da fase de antecipação, e a estratégia de Autocontrole, representativa da fase de desempenho.

\section{CONCLUSÃO}

Concluindo, a dicotomia básica entre aprendizagem heterorregulada (determinada pelas contingências externas) e aprendizagem autorregulada (determinada pelo próprio indivíduo, com base em uma variedade de estratégias), apontada para a aprendizagem escolar em geral, parece ter igualmente presença no domínio específico, aqui investigado, da aprendizagem do desenho. Em particular, pode concluir-se pela possibilidade de uma replicação, no caso da aprendizagem do desenho, da maioria das estratégias autorregulatórias encontradas na literatura para a aprendizagem em geral, mais do tipo conceitual (Costa e Boruchovitch, 2010a, 2010b; Encarnacao e Vázquez-Bernal, 2013; Rosário et al., 2005; Zimmerman e 
Martinez-Pons, 1986). Esse paralelismo sugere também que a autorregulação poderá constituir um processo extensível da aprendizagem em geral para a aprendizagem das artes em particular.

No entanto, essas possibilidades devem ser entendidas com cautela, considerando as limitações do estudo aqui apresentado, donde se destaca a reduzida amostra de participantes. Recorde-se que este estudo, de caráter qualitativo e exploratório, não tem a pretensão de generalizar os resultados para a população, mas, antes, para a teoria. Outra limitação se prende com o fato de a avaliação conduzida ter sido centrada na percepção dos participantes sobre o seu processo de aprendizagem do desenho, e não diretamente sobre esse processo. Para além disso, as dificuldades observadas de resposta às questões da entrevista (possivelmente em virtude da ausência de hábitos de autorreflexão) podem acrescer a relativização da validade daquela percepção.

Apesar de tudo, este estudo fornece um contributo à linha de investigação da aprendizagem autorregulada, confirmando-a como igualmente relevante para a pesquisa sobre a aprendizagem da arte e, em particular, do desenho artístico, tão carente de estudos sob essa perspectiva. $\mathrm{O}$ resultado deste estudo, de que a aprendizagem do desenho é potencialmente marcada pela utilização de estratégias autorregulatórias, sugere a possibilidade de que estas sejam desenvolvidas no intuito de uma promoção da aprendizagem da arte. Isso significa que a aprendizagem da arte pode ser perspectivada não só como um produto, mas igualmente como um processo que é possível melhorar.

Esta pesquisa abre, assim, a possibilidade de uma intervenção psicoeducacional com o objetivo de desenvolver as estratégias de autorregulação da aprendizagem da arte no intuito de contribuir para o sucesso e a qualidade daquela aprendizagem ou para a sua autonomia.Em contrapartida,leva a perspectivar a possibilidade de diagnóstico do reportório pessoal de estratégias de aprendizagem da arte, com base no qual se poderá desenvolver planos de intervenção individualizada para desenvolver estratégias autorregulatórias não utilizadas ou adotadas deficientemente. Nesse sentido, as categorias resultantes da análise realizada neste estudo podem servir de base aos itens de um questionário que possa medir quantitativamente as estratégias de autorregulação da aprendizagem do desenho, ou de uma lista de verificação que possa ser utilizada para a sua promoção.

Efetivamente, considera-se a necessidade de estudos futuros quantitativos, com amostras maiores, que avaliem, por questionário, as estratégias de aprendizagem da arte e do desenho. Isso permitiria também investigar como essas estratégias se relacionam com outras variáveis pessoais, contextuais e relativas ao produto daquelas aprendizagens. Finalmente, perspetivam-se estudos futuros que permitam testar os efeitos de intervenções dirigidas ao desenvolvimento de estratégias de aprendizagem da arte em geral e do desenho artístico em particular.

\section{REFERÊNCIAS}

Araújo, R.; Cavalcanti, C.; Figueiredo, E. Motivação para prática musical no ensino superior: três possibilidades de abordagens discursivas. Revista da ABEM, Porto Alegre, v. 18, n. 24 , p. $34-44,2010$. 
BAKeman, R.; Gottman, J. Observing interaction: an introduction to sequencial analysis. London: Cambridge University Press, 1986.

BAndura, A. Social foundations of thought and action: a social cognitive theory. Englewood Cliffs, NJ: Prentice-Hall, 1986.

Bandura, A. A evolução da teoria social cognitiva. In: Bandura, A.; Azzi, R.; Polydoro, S. (Orgs.). Teoria social cognitiva: conceitos básicos. Porto Alegre: Artmed Editora, 2008. p. 15-41.

Becker, M. Autorregulação da aprendizagem em matemática: uma experiência com alunos do ensino médio. 2016.107f. Dissertação (Mestrado em Matemática) - Universidade de Brasília, Brasília, 2016.

BIGGs, J. Student approaches to learning and studying. Melbourne: ACER, 1987.

Boruchovitch, E. A identificação e o estudo das variáveis associadas ao fracasso escolar brasileiro. Projeto de Pesquisa (CNPq-Processo n.300162/95-2) - Faculdade de Educação, Departamento de Psicologia Educacional, Universidade de Campinas, Campinas, 1995.

Catterall, J. Conversation and silence: transfer of learning through the arts. Journal for Learning through the Arts, United States: University of California, v. 1, n. 1, p. 1-12, 2005. https://doi.org/10.21977/D91110081

CorrêA, R.; Correia, P. A utilização do mapa conceitual na análise da autorregulação da aprendizagem no ensino de ciências. Enseñanza de las Ciencias: revista de investigación y experiencias didácticas, Barcelona: UAB, n. extra, p. 5.137-5.144, 2017.

Costa, E.; Boruchovitch, E. Compreendendo relações entre estratégias de aprendizagem e a ansiedade de alunos do ensino fundamental de Campinas. Psicologia: Reflexão e Critica, Porto Alegre: UFRGS, v. 17, n. 1, p. 15-24, 2004. http://dx.doi.org/10.1590/ S0102-79722004000100004

Costa, E.; Boruchovitch, E. As estratégias de aprendizagem de alunos repetentes do ensino fundamental. Psicologia em Pesquisa, Juiz de Fora: UFJF, v. 4, n. 1, p. 31-39, 2010a. Costa, E.; Boruchovitch, E.. Motivação para fazer o dever de casa e estudar para uma matéria desinteressante: relato de alunos do ensino fundamental de Campinas-SP. Itinerarius Reflectionis, Jataí: UFG, v. 2, n. 9, s./p., 2010b. https://doi.org/10.5216/rir. v2i9.1100

Currey, M.Daily rituals: how artists work. New York: Alfred A. Knopf, 2013.

DAura, F. Aprendizaje autorregulado y rendimiento académico en estudiantes del ciclo clínico de la carrera de medicina. Revista Electrónica de Investigación Educativa, México: UABC, v. 17, n. 3, p. 28-45, 2015.

Deci, E.; Ryan, R. Motivation, personality, and development within embedded social contexts: an overview of self-determination theory. In: Ryan, R. (Ed.). The Oxford handbook of human motivation. New York, NY: Oxford University Press, 2012.

Eisner, E. Ocho importantes condiciones para la enseñanza y el aprendizaje en las artes visuales. Arte, Individuo y Sociedad, Madrid: UCM, v. 1, p. 46-56, 2002. 
Encarnacao, C.; Vázquez-Bernal, B. Estrategias de autorregulación en el aprendizaje de las reacciones químicas en alumnos del tercero ciclo en Portugal. Enseñanza de las Ciencias: revista de investigación y experiencias didácticas, Barcelona: UAB, n. extra, p. 605-609, 2013.

Finke, R.; Ward, T.; Smith, S. Creative cognition: theory, research, and applications. Cambridge, MA: MIT Press, 1992.

Flores, J. Análises de datos cualitativos: aplicacione a la investigación educativa. Barcelona: PPV, 1994.

GonZÁLEz, M. Aspectos personales que favorecen la autorregulación del aprendizaje en la comprensión de textos académicos en estudiantes universitarios. REDU. Revista de Docencia Universitaria, València: UPV, v. 13, n. 2, p. 17-35, 2015. https://doi. org/10.4995/redu.2015.5436

Gusmão, P. A aprendizagem autorregulada da percepção musical no ensino superior: uma pesquisa exploratória. Opus, Porto Alegre: ANPPOM, v. 17, n. 2, p. 121-140, 2011. Hinojosa, J.; Sanmartí, N. La autorregulación metacognitiva como medio para facilitar la transferencia en mecánica. Revista Eureka sobre Enseñanza y Divulgación de las Ciencias, Cadiz, Andalucía: UCA, v. 12, n. 2, p. 249-263, 2015.

HLA, K.; Phyu, K. A study of self-regulated learning of high school students. Yuoe Research Journal, United States: Yangon University of Education, v. 3, n. 1, p. 1-12, 2011. Horta, P. A microanálise através da entrevista semidirectiva: uma avaliação do ciclo da autorregulação da aprendizagem. 2010.101f. Dissertação (Mestrado em Psicologia) — Universidade de Lisboa, Faculdade de Psicologia, Lisboa, 2010.

Kaplan, J. Teoria da aprendizagem pianistica. Porto Alegre: Editora Movimento, 2009.

Lawson, B. How designers think. The design process demystified. Oxford: Elesevier, 2003.

LEE, Y.; Jiar, Y. Effects of intervention to develop self-regulated learning behaviour in learning history. Sains Humanika, Johor, Malaysia: Universiti Teknologi Malaysia, v. 2, n. 4, p. 239-245, 2014. https://doi.org/10.11113/sh.v2n4.493

Miles, M.; Huberman, A. Qualitative data analysis: and expanded sourcebook. Thousand Oaks, CA: Sage Publications, 1994.

Monereo, C. et al. Estrategias de enseñanza y aprendizaje: formación del profesorado y aplicación en la escuela. Barcelona: Grão Editorial, 1995. (Colección El Lápiz).

Padilla, R. El dibujo del natural: la época de la postacademia. Madrid: Ediciones Akal, 2007.

Paris, S.; Winograd, P. How metacognition can promote academic learning and instruction. In: Jones, B.; Idol, L. (Orgs.). Dimensions of thinking and cognitive instruction. Hillsdale, N J: Erlbaum, 1990.

Pintrich,P.The role of goal orientation in self-regulated learning.In: Boekaerts, M.; Pintrich, P.; Zeidner, M. (Eds.). Handbook of self-regulation. San Diego, CA: Academic Press, 2000.

Ribeiro, I. Promover a consciência metacognitiva e a autorregulação das estratégias de aprendizagem para aumentar a qualidade da aprendizagem. 2013. 112f. Dissertação (Mestrado em Psicologia) — Universidade de Lisboa, Lisboa, 2013. 
RosÁrio, P. et al. El aprendizaje escolar examinado desde la perspectiva del "modelo 3p" de J. Biggs. Psicothema, Oviedo: Universidad de Oviedo, v. 17, n. 1, p. 20-30, 2005. RosÁrio, P. et al. Processos de autorregulação da aprendizagem em alunos com insucesso no $1^{\circ}$ ano de universidade. Psicologia Escolar e Educacional, São Paulo: ABRAPEE, v. 14, n. 2, p. 349-358, 2010. http://dx.doi.org/10.1590/S141385572010000200017

Roux, R.; González, E. Estrategias de aprendizaje y su relación con el rendimiento académico en estudiantes de una escuela privada de educación media superior. Revista Actualidades Investigativas en Educación, Costa Rica: UCR, v. 15, n. 1, p. 1-16, 2015. 10.15517/AIE.V15I1.17731

Sepulveda-Vildosola, A.; Carrada-Legaria, S.; Reyes-Lagunes, I. Motivación y estrategias de aprendizaje en residentes de pediatría. Gaceta Médica de México, México: Academia Nacional de Medicina de México, v. 15, n. 4, p. 477-484, 2015.

Silva, A. L.; Sá, I. Saber estudar e estudar para saber. Porto: Porto Editora, 1997.

Silva, A. L.; Sá, I.; Duarte, A. M.; Simão, A. M. V. V. Aprendizagem autorregulada pelo estudante: perspectivas psicológicas e educacionais. Porto: Porto Editora , 2004.

Sımão, A. M. V. V. O conhecimento estratégico e a autorregulação da aprendizagem: implicações em contexto escolar. In: Silva, A. L.; Sá, I.; Duarte, A. M.; Simão, A. M. V. V. (Eds.). Aprendizagem autorregulada pelo estudante: perspectivas psicológicas e educacionais. Porto: Porto Editora, 2004.

Soтo, P.; Macías, F. Experiencia educativa en arte visual diseñada bajo un modelo de autorregulación del aprendizaje con estudiantes universitarios. Revista Mexicana de Investigación Educativa, México: COMIE, v. 16, n. 49, p. 597-624, 2011.

Sousa, B. Autorregulação da aprendizagem da língua portuguesa no $1^{\circ}$ ciclo: investigação através de diários. 2015. 49f. Dissertação (Mestrado em Psicologia) — Universidade de Lisboa, Lisboa, 2015.

Teixeira, A. Estratégias de autorregulação na aprendizagem em história: estudo no $2^{\circ}$ CEB 2004. 153f. Dissertação (Mestrado em Psicologia) — Universidade do Minho, Braga, 2004.

Teixeira, A.; Alliprandini, P. Intervenção no uso de estratégias de aprendizagem diante de dificuldades de aprendizagem. Psicologia Escolar e Educacional, São Paulo: ABRAPEE, v. 17, n. 2, p. 279-288, 2013. http://dx.doi.org/10.1590/S141385572013000200010

WAGENER, B. L'autorégulation conjointe de la cognition et des émotions: quel impact sur les apprentissages? Voix Plurielles, St. Catharines, ON: Brock University, v. 12, n. 1, p. 82-103, 2015. https://doi.org/10.26522/vp.v12i1.1176

Winner, E.; Hetland, L. The arts and academic achievement: what the evidence shows. Journal of Aesthetic Education, Illinois: University of Illinois, v. 102, n. 5, p. 3-6, 2001. https://doi.org/10.1080/10632910109600008

Zimmerman, B. J. A social cognitive view of self-regulated academic learning. Journal of Educational Psychology, United States: APA, v. 81, n. 3, p. 329, 1989. 
Zimmerman, B. J. Developing self-fulfilling cycles of academic regulation: an analysis of exemplary instructional models. In: Schunk, D.; Zimmerman, B. (Eds.). Self-regulated learning: from teaching to self-reflective practice. New York: The Guilford Press, 1998. p. 1-19.

Zimmerman, B.J.Attaining self-regulation: a social cognitive perspective. In: Boekaerts, M.; Pintrich, P.; Zeidner, M. (Eds.). Handbook of self-regulation. San Diego: Academic Press, 2000. p. 13-39. https://doi.org/10.1016/B978-012109890-2/50031-7

Zimmerman, B. J.; Labuhn A. Self-regulation of learning: process approaches to personal development. In: Harris, K.; Graham, S.; Urdan, T. (Eds.). The educational psychology handbook: theories, constructs, and critical issues. Washington, DC: American Psychological Association, 2012. p. 399-425. http://dx.doi.org/10.1037/13273-014

Zimmerman, B. J.; Martinez-Pons, M. Development of a structured interview for assessing student use of self-regulated learning strategies. American Educational Research Journal, Thousand Oaks, CA, v. 23, n. 4, p. 614-628, 1986. 


\section{Anexo - Roteiro de entrevista da aprendizagem autorregulada do desenho ${ }^{2}$}

1. Estratégias de aprendizagem utilizadas na sala de aula

Vamos imaginar que você está em uma aula de desenho. Você tem alguma maneira que possa the ajudar a aprender o que está sendo dado nessa aula?

\section{Compreensão de conteúdo}

Alguns alunos às vezes percebem que a aprendizagem do desenho se torna difícil e que eles não estão conseguindo aprender. Isso acontece com você?

\section{Estratégias de planificação}

Vamos imaginar que você tem a tarefa de fazer um desenho sem a ajuda do seu professor. Você tem alguma maneira ou método que possa lhe ajudar a planejar essa tarefa?

\section{Estratégias de aprendizagem em casa}

Vamos imaginar que você tenha que aprender desenho em casa. Você tem algum modo ou estratégia que possa the ajudar a aprender melhor?

\section{Estratégias para a avaliação}

Como sabe, a aprendizagem do desenho na faculdade é sujeita à avaliação, que pode ser processual ou ocorrer em momentos especiais. Como você se prepara para a avaliação?

\section{Estratégias de motivação}

Por vezes os alunos não fazem suas tarefas de desenho porque têm uma porção de outras coisas que eles consideram mais importantes, como estudar para outras disciplinas. O que você faz para se motivar e conseguir realizar as tarefas nessas condições?

\section{Decorar}

Às vezes você precisa reter algo para aprender desenho, você tem alguma maneira que possa lhe ajudar nessa situação?

\section{Administração do tempo}

Quando você tem que entregar uma tarefa de desenho no dia seguinte e percebe que não vai dar tempo de fazer tudo que precisa, o que você faz?

\section{Provas}

Quando você está desenhando sob avaliação e percebe que tem dificuldades, o que você costuma fazer?

\section{Correção de desempenho}

Quando você recebe a avaliação do seu desempenho em um desenho, o que você costuma fazer, se o seu desenho não correspondeu aos objetivos propostos?

\section{Motivação para estudar}

Por vezes os alunos acham que aquilo que estão aprendendo no desenho é chato. Isso acontece com você? Você tem alguma maneira de fazer aquilo ficar mais agradável? O que você faz?

\section{Estratégias de organização do ambiente}

Você costuma estudar desenho em qualquer lugar ou você procura um local que lhe ajude a se concentrar mais e a aprender melhor? 


\section{Anexo - Continuação}

\section{Autoavaliação}

Quando você acaba de fazer uma tarefa de desenho, você faz alguma espécie de revisão do seu trabalho?

\section{Autoavaliação}

Quando você está desenhando, você faz alguma coisa para ter certeza de que está realizando o trabalho de maneira adequada?

\section{SOBRE OS AUTORES}

Luiz Gustavo Lima Freire é doutor em psicologia da educação pela Universidade de Lisboa (Portugal). Professor do Centro Universitário dos Guararapes (UNIFG).

E-mail: gstv.psi@gmail.com

António Manuel Duarte é doutor em psicologia da educação pela Universidade de Lisboa (Portugal). Professor da mesma instituição.

E-mail:amduarte@psicologia.ulisboa.pt

Recebido em 27 de fevereiro de 2018 Aprovado em 04 de junho de 2018 